\title{
Topoisomer Differentiation of Molecular Knots by FTICR MS: Lessons from Class II Lasso Peptides
}

\author{
Séverine Zirah, ${ }^{1,4}$ Carlos Afonso, ${ }^{2}$ Uwe Linne, ${ }^{3}$ Thomas A. Knappe, ${ }^{3}$ \\ Mohamed A. Marahiel, ${ }^{3}$ Sylvie Rebuffat, ${ }^{1}$ Jean-Claude Tabet ${ }^{2}$ \\ ${ }^{1}$ National Museum of Natural History, Communication Molecules and Adaptation of Micro-organisms, FRE 3206 \\ CNRS - MNHN, CP 54, 57 rue Cuvier, F-75005 Paris, France \\ ${ }^{2}$ Parisian Institute of Molecular Chemistry, UMR 7201 CNRS - University Pierre \& Marie Curie, 4 place Jussieu, F-75005 \\ Paris, France \\ ${ }^{3}$ Department of Chemistry, Philipps-University, Fb. 15-Chemie, Hans-Meerwein-Straße, 35032 Marburg, Germany \\ ${ }^{4}$ Molécules de communication et adaptation des micro-organismes, FRE 3206 CNRS/Muséum National d'Histoire Naturelle, \\ CP 54, 57 rue Cuvier, 75005 Paris, France
}

\begin{abstract}
Lasso peptides constitute a class of bioactive peptides sharing a knotted structure where the $\mathrm{C}$-terminal tail of the peptide is threaded through and trapped within an $\mathrm{N}$-terminal macrolactam ring. The structural characterization of lasso structures and differentiation from their unthreaded topoisomers is not trivial and generally requires the use of complementary biochemical and spectroscopic methods. Here we investigated two antimicrobial peptides belonging to the class II lasso peptide family and their corresponding unthreaded topoisomers: microcin J25 (MccJ25), which is known to yield two-peptide product ions specific of the lasso structure under collisioninduced dissociation (CID), and capistruin, for which CID does not permit to unambiguously assign the lasso structure. The two pairs of topoisomers were analyzed by electrospray ionization Fourier transform ion cyclotron resonance mass spectrometry (ESI-FTICR MS) upon CID, infrared multiple photon dissociation (IRMPD), and electron capture dissociation (ECD). CID and ECD spectra clearly permitted to differentiate MccJ25 from its non-lasso topoisomer MccJ25-Icm, while for capistruin, only ECD was informative and showed different extent of hydrogen migration (formation of $c / z$ from $c / z_{0}$ ) for the threaded and unthreaded topoisomers. The ECD spectra of the triply-charged MccJ25 and MccJ25-Icm showed a series of radical $b$-type product ions $\left(b^{\prime} \bullet_{n}\right)$. We proposed that these ions are specific of cyclic-branched peptides and result from a dual $c / z \bullet$ and $y / b$ dissociation, in the ring and in the tail, respectively. This work shows the potentiality of ECD for structural characterization of peptide topoisomers, as well as the effect of conformation on hydrogen migration subsequent to electron capture.
\end{abstract}

Key words: Lasso peptides, Topoisomers, ECD, FTICR, Hydrogen migration, MccJ25, Capistruin, $b^{\prime} \cdot$-type ions

Séverine Zirah and Carlos Afonso have contributed equally to the work.

Electronic supplementary material The online version of this article (doi:10.1007/s13361-010-0028-1) contains supplementary material, which is available to authorized users.

Correspondence to: Séverine Zirah; e-mail: szirah@mnhn.fr

\section{Introduction}

$\mathrm{M}$ olecular knots constitute original structural motifs in biomolecules and fascinating topological objects [1, 2]. Bioactive peptides with knotted structures, such as lasso peptides and cyclotides, share a knot topologically defined 
by covalent crosslinks (lactam ring and/or cysteine bridges) and display very compact and stable structures. Such structures therefore constitute valuable scaffolds with potential applications in peptide-based drug design [3, 4]. Lasso peptides form a class of bacteria-produced bioactive peptides with a threaded structure involving an 8/9-residue ring resulting from an amide bond between the $N$-terminus and the carboxylic group of an Asp/Glu residue, crossed by the $C$-terminal tail [5]. They form three classes depending on the absence (class II) or presence of one (class III) or two (class I) disulfide bridges that further stabilize the structure [6]. They exhibit a variety of biological activities, involving enzyme inhibition, receptor antagonism, antimicrobial or anti-HIV activities (see Table 1 for class II lasso peptides) [5, 7-12]. Their compact structure confers to lasso peptides a great resistance to denaturing conditions and certain proteases. The structural characterization of lasso structures and differentiation from their unthreaded topoisomers is not trivial and generally requires the use of complementary biochemical and spectroscopic methods.

Microcin J25 (MccJ25) is a lasso peptide secreted by Escherichia coli AY25 that exerts potent antibacterial activity against Escherichia and Salmonella species, through import in the target bacteria upon interaction with the ironsiderophore receptor FhuA [13] and inhibition of the RNA polymerase [14]. Its $C$-terminal (18-21 SFYG) segment is sterically entrapped into the macrolactam ring by F19 and Y20 located on each side of the ring. Cleavage within the Y9-I18 region, either in solution by enzymatic cleavage or acidic hydrolysis, or in the gas phase upon collision-induced dissociation (CID), generates two peptide complexes associated through the steric hindrance provided by the side chains of F19 and Y20 [15-17]. A particular gas-phase fragmentation pattern upon CID, compared with the corresponding synthetic peptide encompassing a macrolactam ring, has also been reported for the lasso peptide RES 701-1 [18], but generally only NMR could unambiguously characterize the threading of the $C$-terminal tail into the macrolactam rings $[7,8,19]$. Capistruin is a lasso peptide secreted by Burkholderia thailandensis E264 discovered by genome mining, which exhibits antibacterial activities against closely related Burkholderia and Pseudomonas species [8]. It showed a low overall fragmentation pattern upon CID. However, no two-peptide complexes unequivocally proving a lasso structure were observed [8]. Comparison of the threedimensional structures of MccJ25 and capistruin shows that

Table 1. Sequences and biological activities of type-II lasso peptides ${ }^{\mathrm{a}}$

\begin{tabular}{|c|c|c|c|}
\hline Sequence & $\begin{array}{l}\text { Producing } \\
\text { bacteria }\end{array}$ & $\begin{array}{l}\text { Biological } \\
\text { activity }\end{array}$ & Reference \\
\hline 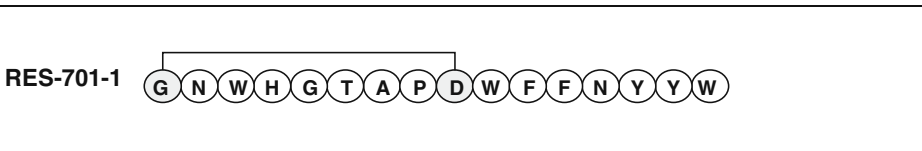 & $\begin{array}{l}\text { Streptomyces } \\
\text { sp. RE-701 }\end{array}$ & $\begin{array}{l}\text { Endothelin type B } \\
\text { receptor selective } \\
\text { antagonist }\end{array}$ & [9] \\
\hline Anantin $^{b}$ GEDGWGNODOFGMYSGDF & $\begin{array}{l}\text { Streptomyces } \\
\text { coerulescens }\end{array}$ & $\begin{array}{l}\text { Atrial natriuretic } \\
\text { factor antagonist }\end{array}$ & [12] \\
\hline Propeptin $^{\text {b }}$ GPPWWDYBDDFGGWOFDSP & $\begin{array}{l}\text { Microbispora } \\
\text { sp. SNA-115 }\end{array}$ & $\begin{array}{l}\text { Prolyl } \\
\text { endopeptidase } \\
\text { inhibitor }\end{array}$ & [11] \\
\hline 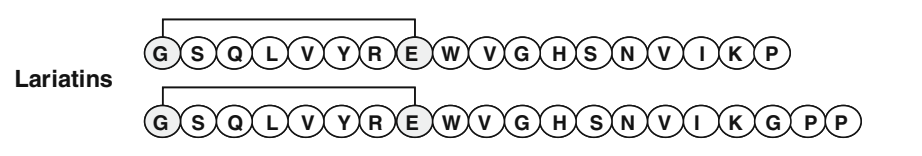 & $\begin{array}{l}\text { Rhodococcus } \\
\text { sp. K01- } \\
\text { B0171 }\end{array}$ & Antimycobacterial & [7] \\
\hline 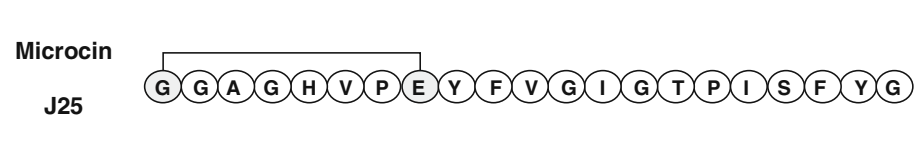 & $\begin{array}{l}\text { Escherichia } \\
\text { coli AY25 }\end{array}$ & $\begin{array}{l}\text { Antibacterial } \\
\text { RNA polymerase } \\
\text { inhibitor }\end{array}$ & {$[10,15,17]$} \\
\hline 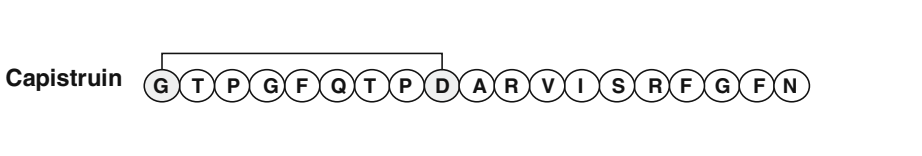 & $\begin{array}{c}\text { Bhurkholderia } \\
\text { thailandensis } \\
\text { E264 }\end{array}$ & Antibacterial & [8] \\
\hline
\end{tabular}

\footnotetext{
a The sequences are represented unthreaded for clarity.

${ }^{\mathrm{b}}$ The lasso structure (threading of the tail into the macrolactam ring) is hypothesized but has not been shown.
} 
MccJ25 displays a large loop involving a short antiparallel $\beta$-sheet and a very short $C$-terminal segment below the ring, while capistruin displays a very tight loop and a much longer $C$-terminal segment below the ring (Figure 1). In both peptides, the steric entrapping of the $C$-terminal tail in the macrolactam ring relies on the presence of a bulky amino acid located below the ring: Y20 for MccJ25 [20] and R15 for capistruin [21]. In addition, both peptides contain a bulky amino acid located above the ring: F19 for MccJ25 and R11 for capistruin. However, in capistruin the two bulky amino acids, R11 and R15, are not adjacent.

Fourier transform ion cyclotron resonance (FTICR) mass spectrometry has shown a great capability for the analysis of peptides and proteins, in particular for the characterization of post-translational modifications [22]. The main advantages of this method are the high resolution together with the multiple ion activation modes available, such as (1) CID in the external collision cell or in the ICR cell through sustained off resonance irradiation (SORI) under CID conditions [23], (2) infrared multiphoton dissociation (IRMPD) [24], and (3) for positive ions, electron-capture dissociation (ECD) $[25,26]$. The latter has revealed particularly powerful for the structural characterization of peptides and proteins [27], given the ability to cleave the peptide backbone while leaving intact labile side-chain modifications, or without disrupting the noncovalent interactions involved in the three-dimensional structure of proteins $[28,29]$. In order to develop an analytical method to unambiguously characterize topoisomeric peptides by mass spectrometry, the fragmentation patterns MccJ25, capistruin, and their corresponding non-lasso topoisomers
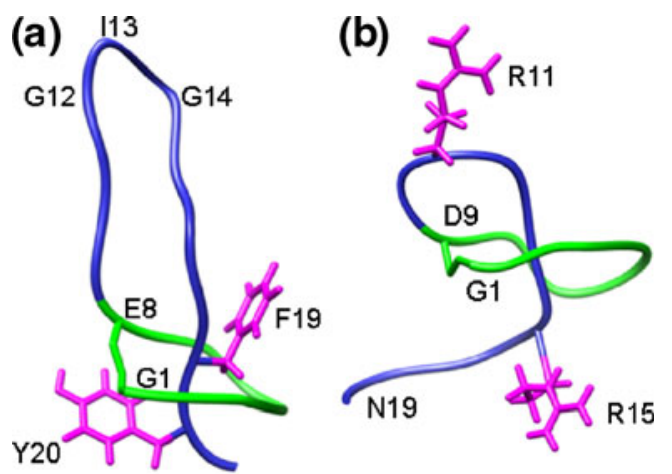

(c)

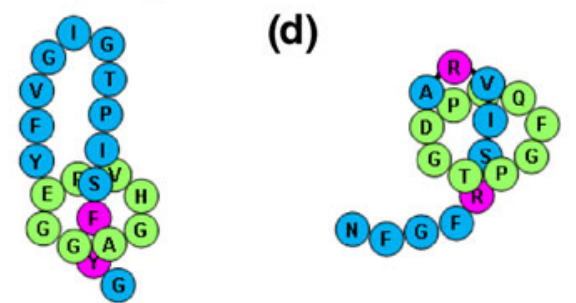

Figure 1. Three-dimensional structures of MccJ25 [17] (a) and capistruin [8] (b). Scheme representing MccJ25 (c) and capistruin (d), showing the macrolactam ring in green, the $C$ terminal tail in blue and the bulky residues located above and below the macrolactam ring in magenta
MccJ25-lcm and capistruin-lcm were analyzed by ESI-FTICR-MS upon CID, SORI CID, IRMPD, and ECD.

\section{Experimental}

\section{Peptides}

MccJ25 was produced from a culture of E. coli MC4100 harboring the plasmid pTUC202, cultivated for $16 \mathrm{~h}$ in M63 medium supplemented with $1 \mathrm{mg} / \mathrm{mL}$ vitamin $B_{1}, 0.02 \%$ $\mathrm{MgSO}_{4}, 0.02 \%$ glucose and $1 \mathrm{~g} / \mathrm{L}$ casamino acids. MccJ25 was purified from the culture supernatant by solid phase extraction on a SepPak C18 $35 \mathrm{cc}$ cartridge and by semi-preparative RPHPLC on a C18 $\mu$ Bondapak column $(300 \mathrm{~mm} \times 3.9 \mathrm{~mm}$, $10 \mu \mathrm{m}$, Waters, Saint-Quentin En Yvelines, France).

Capistruin was produced from a culture of Burkholderia thailandensis E264 incubated for $24 \mathrm{~h}$ at $42^{\circ} \mathrm{C}$ in $\mathrm{M} 20$ medium containing gentamycin $(8 \mu \mathrm{g} / \mathrm{mL})$, as described previously [8]. The purification was carried out by solid phase extraction followed by preparative RP-HPLC [8]. The synthetic cyclic branched peptides (MccJ25-lcm and capistruin-lcm) and linear MccJ25 were obtained from Genepep (Montpellier, France).

\section{FTICR Mass Spectrometry}

Mass spectrometry experiments were performed on a hybrid Qh-FTICR (Bruker Daltonics, Bremen, Germany) equipped with an off-axis Apollo II ESI source. Ionization was performed in positive mode with an ESI voltage of $3500 \mathrm{~V}$. The capillary exit was set to $300 \mathrm{~V}$. Skimmers I and II were set to 40 and $8 \mathrm{~V}$, respectively. A syringe pump was used to infuse the peptide solution $\left(10 \mu \mathrm{M}\right.$ in $\mathrm{H}_{2} \mathrm{O}$ / $\mathrm{CH}_{3} \mathrm{CN}+0.1 \%$ (vol/vol) formic acid) with a gas-tight syringe at a flow rate of $120 \mu \mathrm{L} / \mathrm{h}$. CID experiments were performed in the hexapole collision cell or upon SORI CID in the ICR cell using argon as collision gas $(30 \mathrm{~ms}$ pulse from a 7 mbar argon reservoir). For SORI CID, the ions were activated using a $-600 \mathrm{~Hz}$ frequency offset for $250 \mathrm{~ms}$ with a $2.0 \mathrm{~V}_{p-p}$. A pumping delay of $2 \mathrm{~s}$ was applied prior to ion analysis. IRMPD was performed with a $25 \mathrm{~W} \mathrm{CO}_{2}$ laser (Synrad, Mukilteo, WA, USA) operating at $10.6 \mu \mathrm{m}$. Photon irradiation energy was set at $60 \%$ of full laser power. The irradiation time was set to $300 \mathrm{~ms}$ and sidekick and trapping voltages were tuned in order to obtain a good ion cloud overlapping. When necessary, $\mathrm{MS}^{3}$ experiments were conducted using CID in the hexapole collision cell in conjunction with either SORI CID or IRMPD. ECD experiments were performed with an indirectly heated hollow cathode set to $1.7 \mathrm{~A}$. Electrons emitted during $0.1 \mathrm{~s}$ were injected into the ICR cell with a $1.2 \mathrm{~V}$ bias and ECD lens of $20 \mathrm{~V}$. The major specific setup parameters are indicated in the figure legends. All ESI mass spectra were acquired with XMASS (ver. 6.1; Bruker Daltonics) in broadband mode from $\mathrm{m} / \mathrm{z} 100$ to $\mathrm{m} / \mathrm{z} 2200$. The image signal was amplified and digitized using $512 \mathrm{~K}$ data point 
resulting in the recording of a $0.24 \mathrm{~s}$ time domain signal, which was transformed into the corresponding frequency domain by Fourier transform (one zero fill and Sine-bell apodization).

\section{Nomenclature of the Product Ions}

To avoid any ambiguity, the nomenclature introduced by Roepstorff and Fohlman [30], further modified by Biemann [31], was adopted to describe the product ions. For internal fragment ions, the general presentation form is $\left(b_{\mathrm{r}} y_{\mathrm{s}}\right)_{(\mathrm{r}+\mathrm{s}-\mathrm{t})}$, where $r, s$, and $t$ indicate the number of amino acids cleaved counting from the $\mathrm{N}$-terminus, the number of amino acids cleaved from the $C$-terminus and the total number of amino acid residues in the peptide, respectively. The subscript $(r+s$ $-t$ ) thus accounts for the number of residues in the internal fragment. As an example, the internal fragment GIGTPI of MccJ25 has $r=17, s=10$, and $t=21$ and is denoted $\left(b_{17} y_{10}\right)_{6}$. The product ions resulting from fragmentation in the loop of the lasso structure and where the $C$-terminal tail is threaded into the macrolactam ring were denoted $\left[\left(b_{\mathrm{r}}\right)^{*}\left(y_{\mathrm{s}}\right)\right]$, where $r$ and $s$ indicate the number of amino acids in the noncovalently bound $N$-terminal and $C$-terminal peptide segments. As an example, the product ion [MccJ25 $\left\{\right.$ GIGTPI\}] is denoted $\left[\left(b_{11}\right)^{*}\left(y_{4}\right)\right]$. For ECD fragmentations, similar notation using $c / z$ codes were used. The charge states were omitted for singly-charged species and indicated for doubly- and triply-charged species.

\section{Results and Discussion}

\section{Fragmentation Patterns of MccJ25 and Capistruin and Their Non-Lasso Topoisomers Generated by CID, SORI CID, and IRMPD}

The CID spectra of MccJ25, capistruin, and their corresponding synthetic non-lasso topoisomers, $\mathrm{Mcc} J 25-\mathrm{lcm}$, and capistruin-lcm, which contain the macrolactam ring but without steric entrapping of the $C$-terminal tail inside, were obtained through CID in the external collision cell or SORICID in the ICR cell. The spectra provided similar information, therefore only SORI CID spectra are presented, since these experiments permit to avoid $\mathrm{m} / \mathrm{z}$ discrimination during the ion transfer between the collision cell and the ICR cell.

The SORI CID spectrum of the $[\mathrm{M}+3 \mathrm{H}]^{3+}$ species of MccJ25 ( $\mathrm{m} / \mathrm{z}$ 703.0) showed a complicated fragmentation pattern, involving two competitive dissociation processes (Figure 2a, supplementary Table S1). On the one hand, extensive fragmentations in the Y9-I17 loop were detected, yielding typical two-peptide product ions resulting from the steric entrapping of the (SFYG) $C$-terminal segment in the macrolactam ring, as already reported $[15,17]$. The main product ions consisted of $\left[\left(b_{16}\right) *\left(y_{4}\right)\right]^{3+, 2+}$ and $\left[\left(b_{15}\right)^{*}\left(y_{4}\right)\right]^{3+, 2+}$, with consecutive $\mathrm{H}_{2} \mathrm{O}$ and $\mathrm{CO}$ neutral losses, together with $\left[\left(b_{11}\right) *\left(y_{6}\right)\right]^{2+}$ and $\left[\left(b_{11}\right) *\left(y_{4}\right)\right]^{2+}$. Several internal product ions, complementary to the two-peptide product ions, were also detected: $\left(b_{15} y_{10}\right)_{4},\left(b_{17} y_{10}\right)_{6},\left(b_{19} y_{8}\right)_{6},\left(b_{16} y_{9}\right)_{4} \ldots$ On the second hand, complementary $y / b$ series corresponding to fragmentations in the $C$-terminal tail were detected. They consisted of the $b_{8}, b_{9}, b_{10}, b_{11}^{1+, 2+}, b_{12}, b_{13}{ }^{1+, 2+}, b_{19}{ }^{2+}$, $b_{20}{ }^{3+}$ series, together with the $y_{2}, y_{3}, y_{4}$, and $y_{6}$ series (supplementary Table S1). These series indicated a release of the (SFYG) $C$-terminal segment and therefore that fragmentation in the macrolactam ring occurred. Alternatively, the $b$ series could result from consecutive dissociation from $\mathrm{b}_{20}{ }^{3+}$. Indeed, the loss of the $C$-terminal G21 residue may release the $C$-terminal tail. Finally, consecutive $\mathrm{H}_{2} \mathrm{O}$ and $\mathrm{CO}$ losses from the precursor ion were observed $\left(\left[\mathrm{M}+3 \mathrm{H}-\mathrm{H}_{2} \mathrm{O}\right]^{3+}\right.$ being the base peak of the spectrum). The SORI CID spectrum of the $[\mathrm{M}+2 \mathrm{H}]^{2+}$ species of MccJ25 displayed a weak dissociation profile, with $\left[\mathrm{M}+2 \mathrm{H}-\mathrm{H}_{2} \mathrm{O}\right]^{2+}$ as base peak (data not shown). The $[\mathrm{M}+3 \mathrm{H}]^{3+}$ species of MccJ25-1cm showed a more specific fragmentation pattern under SORI CID than that of its lasso topoisomer (Figure 2b, supplementary Table S2). As for MccJ25, the base peak was $\left[\mathrm{M}+3 \mathrm{H}-\mathrm{H}_{2} \mathrm{O}\right]^{3+}$ at $m / z$ 697.0. The spectrum was dominated by the $b$ series (singly-charged $b_{9}$ to $b_{13}$, doubly-charged $b_{11}{ }^{2+}$ to $b_{15}{ }^{2+}, b_{17}{ }^{2+}, b_{19}{ }^{2+}$, triplycharged $b_{20}{ }^{3+}$ ). Intense product ions corresponding to one or two $\mathrm{H}_{2} \mathrm{O}$ losses were observed for $b_{17}{ }^{2+}, b_{19}{ }^{2+}$, and $b_{20}{ }^{3+}$. This behavior is most likely due to the presence of the T15 and S18 residues in the vicinity of the charge. The complementary $y_{2}, y_{4}$, $y_{6}, y_{7}$, and $\left[y_{8}-\mathrm{H}_{2} \mathrm{O}\right]$ were also observed. Finally, only few internal product ions were detected, such as $\left[\left(b_{19} y_{8}\right)_{6}-2 \mathrm{H}_{2} \mathrm{O}\right]$ $(\mathrm{m} / \mathrm{z}$ 567.3). As expected, two-peptide product ions were not detected.

The IRMPD spectrum of the $[\mathrm{M}+3 \mathrm{H}]^{3+}$ species of MccJ25 ( $/ z / z$ 703.0) revealed to be less complex than the corresponding SORI CID spectrum (Figure 3a, supplementary Table S3). The spectrum was dominated by the $b$ series ( $b_{8}$ to $b_{13}, b_{16}{ }^{2+}, b_{17}{ }^{2+}$, and $b_{19}{ }^{2+}$ with one or two $\mathrm{H}_{2} \mathrm{O}$ losses). The $y$ series consisted in $y_{2}, y_{3}, y_{4}, y_{6}$. Only few low abundance twopeptide product ions were detected: $\left[\left(b_{16}\right)^{*}\left(y_{4}\right)-\mathrm{H}_{2} \mathrm{O}\right]^{3+, 2+}$ $(m / z 659.3,988.5)$ and $\left[\left(b_{15}\right) *\left(y_{4}\right)-\mathrm{H}_{2} \mathrm{O}\right]^{2+}(m / z$ 939.9). This result contrasts with the SORI CID spectrum where these two peptide ions are produced in high abundance. In addition, several internal product ions were observed, in particular $\left(b_{10} y_{13}\right)_{2}$ and $/$ or $\left(b_{20} y_{3}\right)_{2}(\mathrm{~m} / z 311.1)$, together with $\left(b_{17} y_{10}\right)_{6}-$ $\mathrm{H}_{2} \mathrm{O}(\mathrm{m} / z$ 521.3). This fragmentation pattern illustrates that IRMPD triggers consecutive dissociations that result in multiple neutral losses and in the opening of the macrolactam ring and, therefore, in the release of the entrapped $C$-terminal segment. A similar behavior towards IRMPD was observed for the $[\mathrm{M}+2 \mathrm{H}]^{2+}$ species of MccJ25 (data not shown). The IRMPD spectrum of the $[\mathrm{M}+3 \mathrm{H}]^{3+}$ species of MccJ25-lcm $(\mathrm{m} / \mathrm{z}$ 703.0) showed a $y / b$ fragmentation pattern close to that of MccJ25, but with a significantly higher fragmentation efficiency (Figure 3b, supplementary Table S4). While for the lasso peptide $\mathrm{MccJ} 25$ the precursor ion dominated the IRMPD spectrum, for the non-lasso topoisomer, the $b_{13}$ product ion was the most intense ion. The IRMPD spectra of MccJ25 and MccJ25-lcm were dominated by b/y series corresponding to 

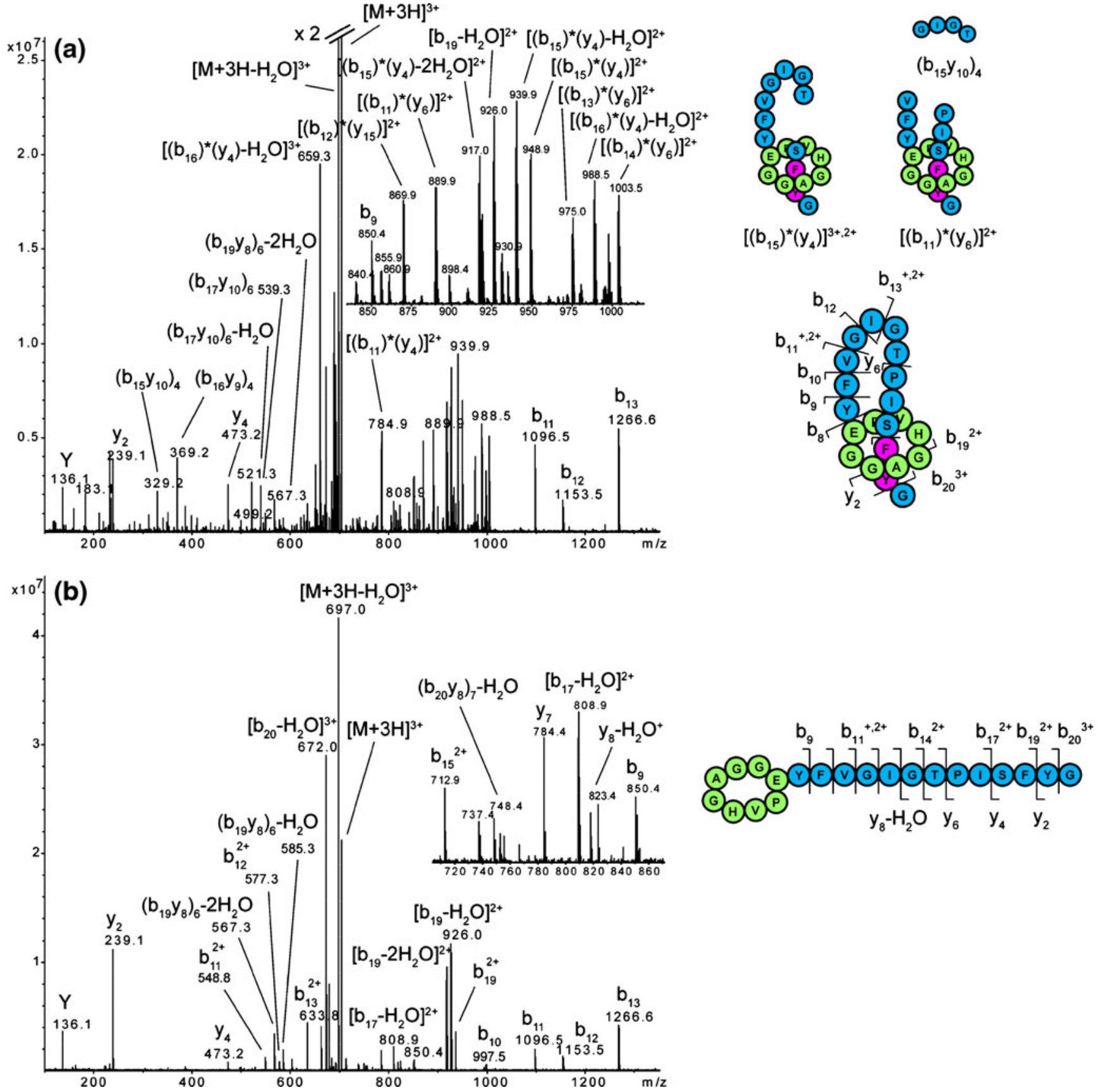

Figure 2. SORI CID spectra of the $[\mathrm{M}+3 \mathrm{H}]^{3+}$ species of (a) MccJ25 and (b) MccJ25-Icm $(\mathrm{m} / \mathrm{z}$ 703.0) recorded with an excitation amplitude of $2 \mathrm{~V}_{p-p}$ and a frequency offset of $-600 \mathrm{~Hz}$. Typical product ions are displayed on the right (the neutral losses are not indicated on the schemes for clarity)

cleavages within the $C$-terminal tail but were fairly different in terms of product ion relative abundance. The $b_{17}$ to $b_{20}$ product ions, corresponding to cleavages within the (SFYG) $C$-terminal segment, were favored for the lactam topoisomer as compared to the lasso. This can be explained by the burring of this segment in the macrolactam ring, which may render the amide groups less accessible for protonation and therefore limits the charge-driven cleavage of the corresponding peptide bonds. Only the minor two-peptide product ions characteristic of the lasso topoisomer, i.e., $\left[\left(b_{16}\right)^{*}\left(y_{4}\right)-\mathrm{H}_{2} \mathrm{O}\right]^{3+, 2+}$ and $\left[\left(b_{15}\right)^{*}\left(y_{4}\right)-\mathrm{H}_{2} \mathrm{O}\right]^{2+}$, could allow to unambiguously distinguish the threaded and unthreaded structures.

SORI CID is an "almost" resonant activation mode where only the precursor ion is effectively activated at its cyclotron frequency. With IRMPD, both the precursor ion and product ions present in the laser beam path are activated, which tend to enhance consecutive dissociations [24]. Thus, the [ $\left(b_{\mathrm{r}}\right)^{*}$ $\left(y_{\mathrm{s}}\right)$ ] two-peptide product ions can dissociate, yielding the conventional $y_{\mathrm{s}}$ or $b_{\mathrm{r}}$ product ions. Consecutive dissociation can also explain the high abundance of some internal 

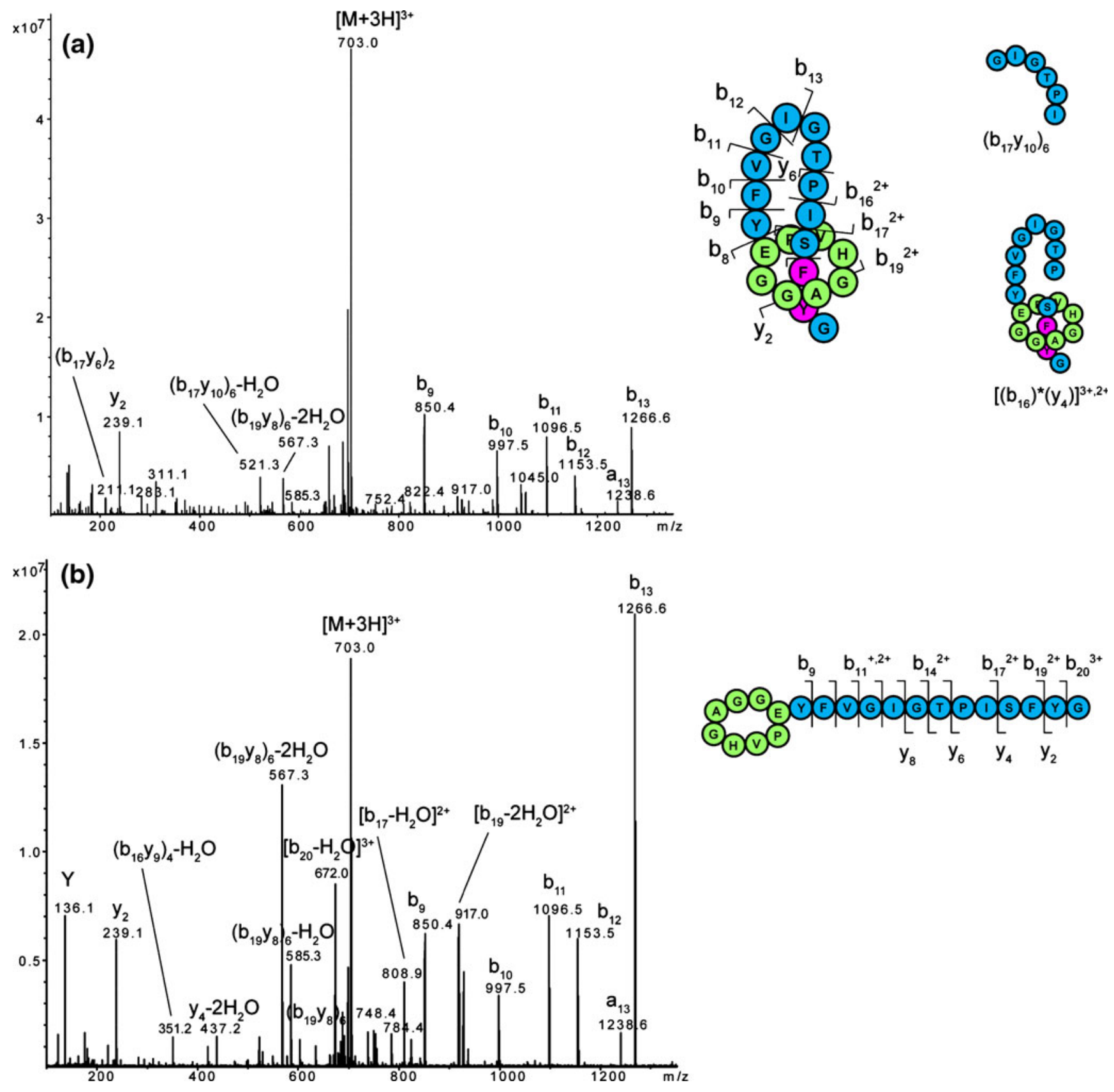

Figure 3. IRMPD spectra of the $[\mathrm{M}+3 \mathrm{H}]^{3+}$ species of (a) MccJ25 and (b) MccJ25-Icm ( $\mathrm{m} / \mathrm{z}$ 703.0). The main product ions are displayed on the right (the neutral losses are not indicated on the schemes for clarity)

product ions such as $\left[\left(b_{19} y_{8}\right)_{6}-2 \mathrm{H}_{2} \mathrm{O}\right](\mathrm{m} / z$ 567.3) (Figures $2 \mathrm{~b}$ and $3 \mathrm{~b}$ ). In this regard, the SORI CID spectrum presents more information about the existence of the lasso form, given the presence of the two-peptide product ions and although in this case the dissociation efficiency is lower as loss of water dominates the product ion spectrum. Thus, resonant activation mode that limits consecutive dissociation is more adequate for the structural characterization of lasso peptides as it allows here to conserve intact two-peptide ions that are a direct proof of the threaded structure.

The SORI CID spectra of the triply-charged capistruin and capistruin-lcm (supplementary Figure S1) were poorly informative, as they showed mainly consecutive $\mathrm{H}_{2} \mathrm{O}$ and $\mathrm{NH}_{3}$ losses from the precursor ion. In addition, capistruin showed very weak $\mathrm{y} / \mathrm{b}$ product ions resulting from cleavages in the $C$-terminal tail. The IRMPD spectra of the triplycharged capistruin and capistruin-lcm (Figure 4) also revealed mainly neutral losses, together with weak $y / b$ series corresponding to cleavages in the $C$-terminal tail that (also affected by extensive neutral losses), and internal product ions. The CID and IRMPD spectra of doubly-charged capistruin and capistruin-lcm displayed mainly neutral losses $\left(\mathrm{H}_{2} \mathrm{O}, \mathrm{NH}_{3} \ldots\right)$ (data not shown). Many common product ions were detected for both topoisomers, but with different 
relative abundance and neutral loss patterns. As an example, the $\left[b_{15}-\mathrm{H}_{2} \mathrm{O}\right]^{2+},\left[b_{16}-\mathrm{H}_{2} \mathrm{O}\right]^{2+},\left[b_{17}-\mathrm{H}_{2} \mathrm{O}\right]^{2+},\left[b_{18}-\mathrm{H}_{2} \mathrm{O}\right]$ ${ }^{2+}$ series dominated the $\mathrm{m} / \mathrm{z} 700-1000$ region in the IRMPD spectrum of capistruin, while this region was dominated by $\left[y_{6}-\mathrm{NH}_{3}\right],\left[y_{7}-\mathrm{NH}_{3}\right],\left[y_{8}-\mathrm{NH}_{3}\right]$ for capistruin-lcm. This trend suggests that the product ions $\left[b_{15}-\mathrm{H}_{2} \mathrm{O}\right]^{2+}$ to $\left[b_{18}-\right.$ $\mathrm{H}_{2} \mathrm{O}^{2+}$, more intense for the lasso topoisomer, correspond to lasso-structured product ions, maintained through R11 and R15 side chains located above and below the ring. Thus, as for MccJ25, the ergodic gas-phase fragmentations of capistruin appear to result in two dissociative processes, (1) the former preserving the lasso structure and yielding the $\left[b_{15}-\mathrm{H}_{2} \mathrm{O}\right]^{2+}$ to $\left[b_{18}-\mathrm{H}_{2} \mathrm{O}\right]^{2+}$ product ions, and (2) the latter yielding $y / b$ series corresponding to cleavages in the $C$ terminal tail, which indicates a previous precursor ion isomerization through opening of the macrolactam ring. However, the short sequence of the loop did not permit to generate two-peptide product ions, which require two bond cleavages in the loop region. Contrary to MccJ25 and its non-lasso topoisomer, the peptides capistruin and capistruin-
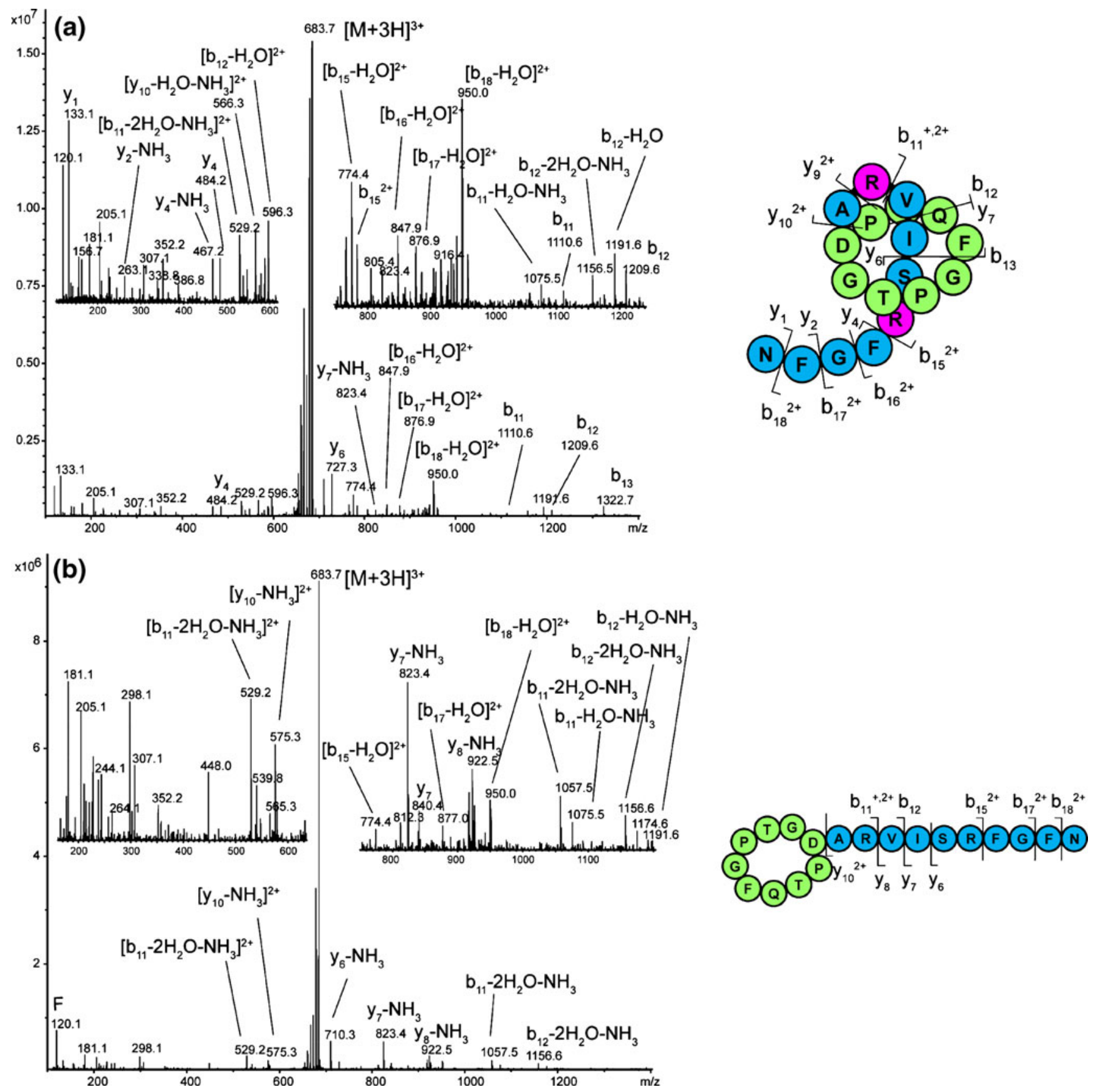

Figure 4. IRMPD spectra of the $[\mathrm{M}+3 \mathrm{H}]^{3+}$ species of (a) capistruin and (b) capistruin-Icm $(\mathrm{m} / \mathrm{z}$ 683.7) recorded with a laser irradiation time of $300 \mathrm{~ms}$. The main product ions are displayed on the right (the neutral losses are not indicated on the schemes for clarity) 
lcm were not distinguished by CID, which was completely dominated by the water loss, but gave different product ion abundance profiles upon IRMPD, which suggests the formation of two-peptide product ions for the lasso topoisomer. This weak fragmentation behavior upon ergodic dissociative processes is attributed to the presence of two arginines (R11 and R15) in the tail region, which most probably sequester the protons. The sequestration of two protons explains the absence of fragmentation for the doubly-charged species, while charge-driven fragmentations occur for the triply-charged species, induced by the extra proton. MccJ25 (one histidine residue in the macrolactam ring) showed an important fragmentation extent, while capistruin (two arginine residues in the tail) yielded only minor sequence product ions. The influence of amino acid sequence (presence of basic residues) and charge state on the extent of ergodic dissociation is a typical illustration of the mobile proton model [32].

\section{Fragmentation Patterns of MccJ25 and Capistruin and Their Non-Lasso Topoisomers Generated by $E C D$}

$X X X$ The ECD spectra of MccJ25 revealed different fragmentations patterns for the $[\mathrm{M}+2 \mathrm{H}]^{2+}$ and $[\mathrm{M}+3 \mathrm{H}]^{3+}$ species (Figure 5a, b). The doubly-charged species generated mainly a $c$ series $\left(c_{17}, c_{18}, c_{19}, c_{20}\right)$, together with two-peptide product ions: $\left[\left(c_{9}\right) *\left(y_{7}\right)-\mathrm{H}_{2} \mathrm{O}\right],\left[\left(c_{10}\right) *\left(y_{7}\right)-\mathrm{H}_{2} \mathrm{O}\right]$ and $\left[\left(c_{11}\right) *\left(y_{7}\right)-\right.$ $\mathrm{H}_{2} \mathrm{O}$ ]. Two relatively intense product ions at $\mathrm{m} / \mathrm{z} 1298.7$ and 1341.7 could not be assigned. The triply-charged species (Figure $5 \mathrm{~b})$ yielded a $y$ series $\left(y_{4}, y_{6}, y_{8}\right)$ and $b$ series $\left(b_{10}, b_{11}\right.$, $b_{13}, b_{15}$ ) showing an unexpected isotopic pattern indicating the presence of $+\mathrm{H} \bullet$ species, noted $b^{\prime}{ }_{n}$ (supplementary Figure S2A). Almost no $c / z \bullet$ ions were detected, with the exception of $c_{18}\left(\mathrm{~m} / z\right.$ 1738.8) and $c_{19}^{2+}(\mathrm{m} / \mathrm{z}$ 943.5). In addition, in a lower extent, two-peptide product ions such as $\left[\left(b_{8}\right) *\left(y_{12}\right)\right]^{2+}$ and the series $\left[\left(c_{8}\right)^{*}\left(y_{4}\right)\right],\left[\left(c_{9}\right)^{*}\left(y_{4}\right)\right]$ and $\left[\left(c_{10}\right)^{*}\left(y_{4}\right)\right]$ were present. Finally, $c / y$ or $b / y$ internal product ions corresponding to tail fragments, i.e., $\left(c_{19} y_{4}\right)_{2},\left(c_{20} y_{4}\right)_{3},\left(c_{19} y_{6}\right)_{4},\left(c_{19} y_{8}\right)_{6}, \ldots$, were detected. The observation of internal product ions by ECD of peptides is unusual, although is has been reported for cyclic peptides [33]. The non-lasso topoisomer MccJ25-lcm, dissociated upon ECD, showed certain trends common to its lasso topoisomer: a $c_{11}$ to $c_{20}$ series for the doubly-charged species (Figure $5 \mathrm{c}$ ), and two competitive dissociation processes for the triply-charged species (Figure 5d): (i) ECD in the ring and consecutive $y / b$ and $b$ ' series and (ii) ECD in the tail yielding a $c / z \bullet$ series. As expected, the two-peptide product ions, characteristic of the lasso structure, were absent. Note that the dissociation efficiency of the charge-reduced species was significantly higher for the non-lasso peptide as compared to the lasso topoisomer. This illustrates the high stability of the lasso structure, as observed by CID.

The almost complete absence of $c / z \bullet$ ions for the $[\mathrm{M}+3 \mathrm{H}]^{3+}$ species of MccJ25 and MccJ25-lcm indicates a particular dissociation pathway. Indeed, electron capture is expected to yield a distonic charge-reduced species $[\mathrm{M}+3 \mathrm{H}] \cdot^{2+}$ that consecutively undergo $c / z \bullet$ cleavage. The formation of $b$-type product ions in ECD experiments has been reported for peptides containing or not basic residues [34-37]. They have been proposed to result from $\mathrm{H} \bullet$ loss from the charge-reduced species and subsequent dissociation of the nonradical species by cleavage of the peptide backbone. Liu and Håkansson proposed that $b$-type ions could also be generated when protonation occurs on the amide groups of the peptide backbone [37]. However, the formation of radical $b^{\prime} \cdot$ through ECD of peptides has never been reported previously. The $b$ ' series and bipeptic product ions permitted to propose a competitive fragmentation pattern involving two isomeric charge-reduced species $[\mathrm{M}+3 \mathrm{H}] \cdot{ }^{2+}(\mathrm{m} / \mathrm{z}$ 1054.5) (Scheme 1). Most likely, the initial ECD occurred within the macrolactam ring, which is consistent with the presence of the basic histidine residue at position 5 (pathway a). The excess of energy in the $[\mathrm{M}+3 \mathrm{H}]{ }^{2+}$ charge-reduced species, due to electron capture, would yield consecutive dissociation involving a conventional $y / b$ cleavage within the $C$-terminal tail ( $b$ series corresponding to $+1 \mathrm{u}$ mass shifts). On the other hand, an initial ECD in the $C$ terminal tail (pathway b) would result in a two-peptide distonic charge-reduced species $[\mathrm{M}+3 \mathrm{H}]{ }^{2+}$, further dissociated into two-peptide and internal product ions. Such pathways result from the presence of both a cyclic and a linear region in the peptide. In order to validate the fragmentation pattern proposed for the formation of $b^{\prime} \cdot$ in MccJ25 and MccJ25-lcm, we performed ECD experiments on the linear variant of MccJ25 (supplementary Figure S2B). The ECD spectrum of the triplycharged linear MccJ25 displayed mainly $c$ and $z \bullet$ product ions. A few $b$-type product ions were detected, such as $b_{13}^{2+}$, observed together with its complementary $y_{8}$. However, the isotopic patterns of $b$-type product ions showed the absence of $b^{\prime}$. species. This result shows that $b^{\prime} \cdot-$-type product ions are generated only for cyclic-branched peptides, which validates the dissociation pathway proposed for MccJ25 and MccJ25$1 \mathrm{~cm}$. The presence of $b$-type product ions in the ECD spectra of linear MccJ25 may result from $\mathrm{H} \cdot$ loss from the distonic charge-reduced species and subsequent dissociation of the nonradical species by cleavage of the peptide backbone, as proposed previously [34-37]. In MccJ25 and MccJ25-lcm, the $b$-type product ions could also be generated from $\mathrm{H} \bullet$ migration from the $b$ ' series.

Multiple backbone cleavages in cyclic peptides have been proposed to result from a free radical reaction cascade in which the $\alpha$-carbon radical formed by electron capture propagates along the peptide backbone by free radical rearrangements $[33,38]$. For MccJ25 and MccJ25-lcm, which are both cyclic-branched peptides, the dissociation appears more complex, with both radical-driven and charge-driven bond cleavages. Such a competition between these two processes has been described recently for arginine-containing peptides, and the absence of basic residues is proposed to favor charge-driven fragmentations while arginine would promote radical-driven fragmentations [39]. The absence of basic residues in the tail of MccJ25 supports this tendency. 

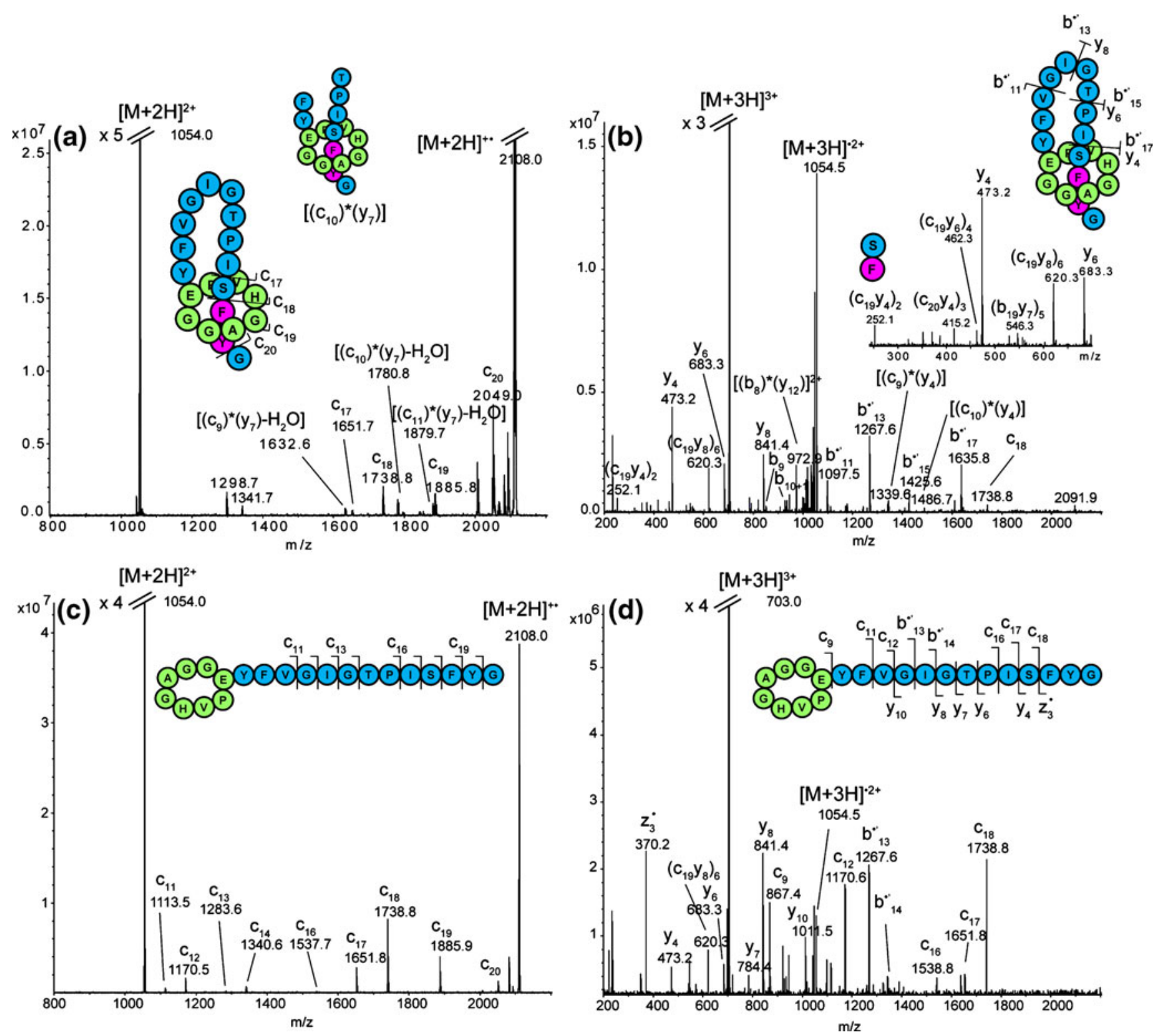

Figure 5. ECD spectra of (a) $[\mathrm{M}+2 \mathrm{H}]^{2+}$ of MccJ25 (m/z 1054.0), (b) $[\mathrm{M}+3 \mathrm{H}]^{3+}$ of MccJ25 $(\mathrm{m} / \mathrm{z} 703.0)$, (c) $[\mathrm{M}+2 \mathrm{H}]^{2+}$ of MccJ25-Icm ( $\mathrm{m} / \mathrm{z}$ 1054.0), and (d) $[\mathrm{M}+3 \mathrm{H}]^{3+}$ of MccJ25- $\mathrm{Icm}(\mathrm{m} / \mathrm{z}$ 703.0), recorded with an ECD bias of $1.2 \mathrm{~V}$ and an irradiation time of $100 \mathrm{~ms}$. The main product ions are displayed (the neutral losses are not indicated on the schemes for clarity)

For both CID and ECD, MccJ25 showed a fragmentation pattern typical of the lasso structure, which permits to assign the lasso character directly, even without a comparison to the non-lasso topoisomer. The formation of the typical twopeptide product ions is particularly favorable for MccJ25, as compared to capistruin given the large size of the loop above the ring and the vicinity of the two bulky amino acids that maintain the $C$-terminal tail entrapped within the ring. Many two-peptide product ions show $\mathrm{y}_{4}$ (SFYG) as minimal entrapped segment.

The dissociation of the $[\mathrm{M}+3 \mathrm{H}]^{3+}$ and $[\mathrm{M}+2 \mathrm{H}]^{2+}$ species of capistruin and its topoisomer capistruin-lcm were also investigated under ECD conditions. The ECD spectra of the $[\mathrm{M}+2 \mathrm{H}]^{2+}$ species of capistruin and capistruin-lcm (supplementary Figure S3) revealed similar fragmentation patterns, with a $c_{16}$ to $c_{18}$ series. The ECD spectrum of the $[\mathrm{M}+3 \mathrm{H}]^{3+}$ species of capistruin (Figure 6a) showed an abundant distonic charge-reduced species $[\mathrm{M}+3 \mathrm{H}] \cdot^{2+}$, together with product ions resulting from neutral losses and a series of $c$ and $z$. ions. The product ion at $\mathrm{m} / \mathrm{z} 996$ corresponded to a loss of $\mathrm{CH}_{5} \mathrm{~N}_{3}$ from the arginine side chain, a usual side chain cleavage in ECD [40]. The $z \bullet_{5}$ to $z^{\circ} 9$ product ions corresponding to cleavages within the tail were fairly abundant. The complementary $c_{11}$ to $c_{18}$ were produced as singly-charged, but also as doubly-charged species for the larger product ions $\left(c_{15}\right.$ to $\left.c_{18}\right) \cdot c_{11}$, the smallest $c$ ion detected, corresponded to the cleavage after $\mathrm{R} 11$. The $z \bullet_{10}$ and $z \bullet_{9}$ product ions were detected, but in very 


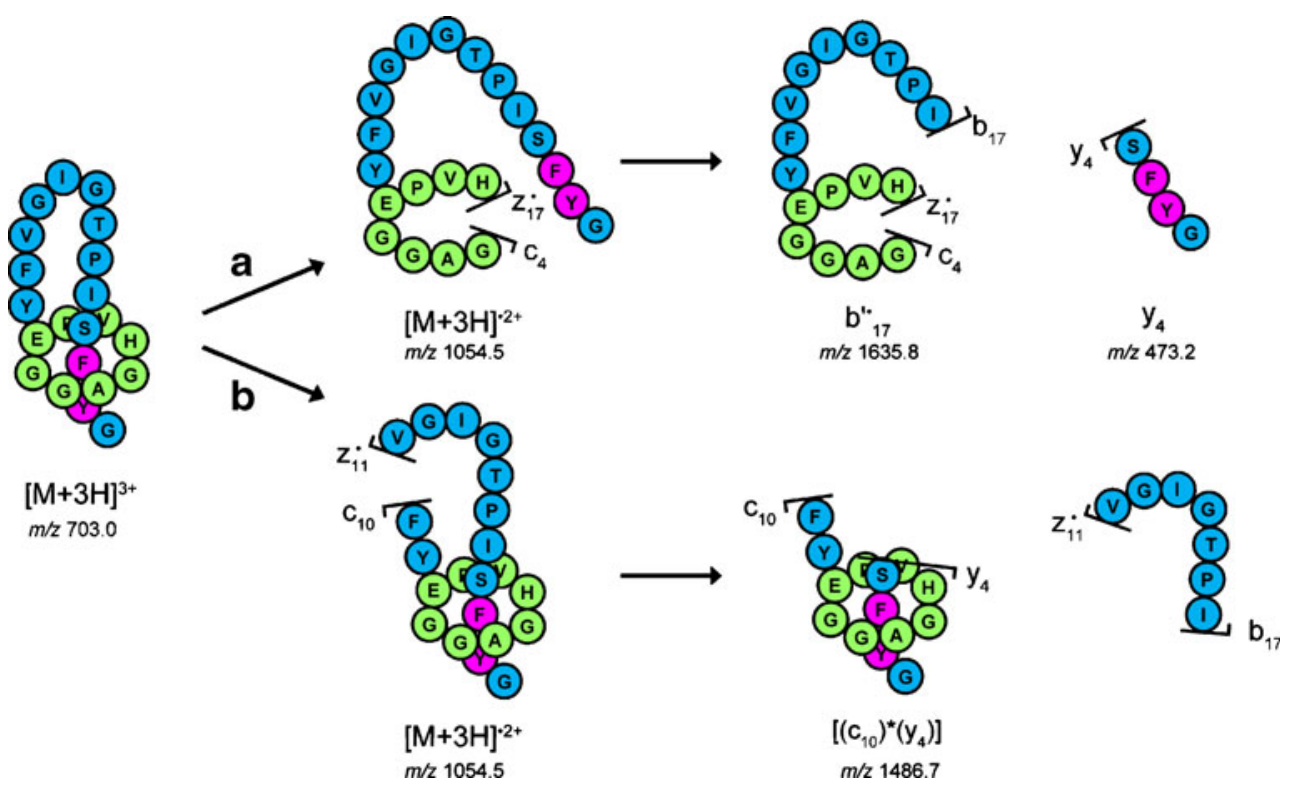

Scheme 1. Pathways proposed for the ECD of the $[M+3 H]^{3+}$ species of MccJ25

low abundance. It should be noted that none of these main cleavages involves dissociation of the ring. This indicates that an initial radical-driven cleavage produced after electron capture occurred within the tail. This is most likely due to the presence of the two basic residues (R11 and R15) in this region, which promotes local $c / z \bullet$ fragmentations in ECD. However, some low abundance product ions were attributed to internal cleavages within the ring. The ECD spectrum of the $[\mathrm{M}+3 \mathrm{H}]^{3+}$ species of the capistruin-lcm topoisomer revealed a similar fragmentation pattern (Figure 6b), but with significant differences in the relative abundance of the product ions. In particular, the $z \bullet_{10}$ and $z \bullet_{9}$ product ions were detected in a much larger abundance as compared to capistruin. This trend suggests that when these cleavages occur for the lasso topoisomer capistruin, the two peptidic entities constitute two-peptide product ions as for MccJ25, due to the steric hindrance provided by R11 and R15 (as already suggested for CID experiments) [8]. In spite of this specific profile of product-ion abundances, no strong evidence of the lasso structure could be detected.

Interestingly, part of the product ions corresponded to $c$ and $z \bullet$, but several $c \bullet$ and $z$ were also detected. Such a trend is relatively frequent during ECD dissociation of peptides and it has been proposed that the $c \cdot$ and $z$ species are produced from $\mathrm{H} \bullet$ migration from $\mathrm{c}$ to $z \bullet$ occurring in a long life $c / z \bullet$ ion/ion complex [41-43]. For capistruin and capistruin-lcm topoisomers, the $c \cdot$ ions revealed particularly abundant for cleavages of the tail nearby the ring. In addition, the relative abundance of $c_{\bullet} / z$ species was strongly reduced for the non-lasso topoisomer. This behavior is illustrated in the spectra enlargement in Figure 6c. In the non-lasso peptide, the normal $c_{11}$ was mainly detected, while a significant amount of $c_{11}$ species was detected for the lasso topoisomer. The same behavior was observed for $z_{9}$, which was mostly detected as $z \bullet 9$ for the capistruin-lcm and as a mixture $z_{5} / z_{9}$ for capistruin. Only a comparison with the non-lasso topoisomer permitted to assign the lasso structure of capistruin, with regard to the relative abundance of the product ions and the extent of hydrogen migration. Therefore, ECD appeared as more powerful in differentiating the two topoisomers capistruin and capistruin-lcm as compared to ergodic dissociation and generated mainly $c / z$ • product ions within the $C$-terminal tail. The absence of $y / b$ product ions suggested that electron capture occurred within the tail and yielded a free radical reaction cascade resulting in multiple backbone cleavages.

\section{Conclusions}

Our data showed that characterization of lasso peptides from gas-phase dissociation can be more or less complex depending on several parameters (Figure 1): (1) the size of the loop above the macrolactam ring, (2) the vicinity of the bulky amino acids in the $C$-terminal tail located above and below the macrolactam ring, and (3) the presence of basic residues within the ring and the tail. The latter limits the chargedriven fragmentation in ergodic dissociation and promotes radical-driven local fragmentations in ECD. ECD appears particularly promising for the characterization of peptide topoisomers, since it provides evidence of the lasso structure for both MccJ25 and capistruin. The presence of two-peptide product ions is the main trend typical of the lasso structure and was clearly observed upon CID and ECD of MccJ25. For capistruin, the absence of double cleavage between D9 and R11 did not permit to detect it. When detected, the twopeptide product ions should generally permit to determine the extent of the $C$-terminal tail that is threaded through the macrolactam ring, from identification of the shorter trapped segment. 

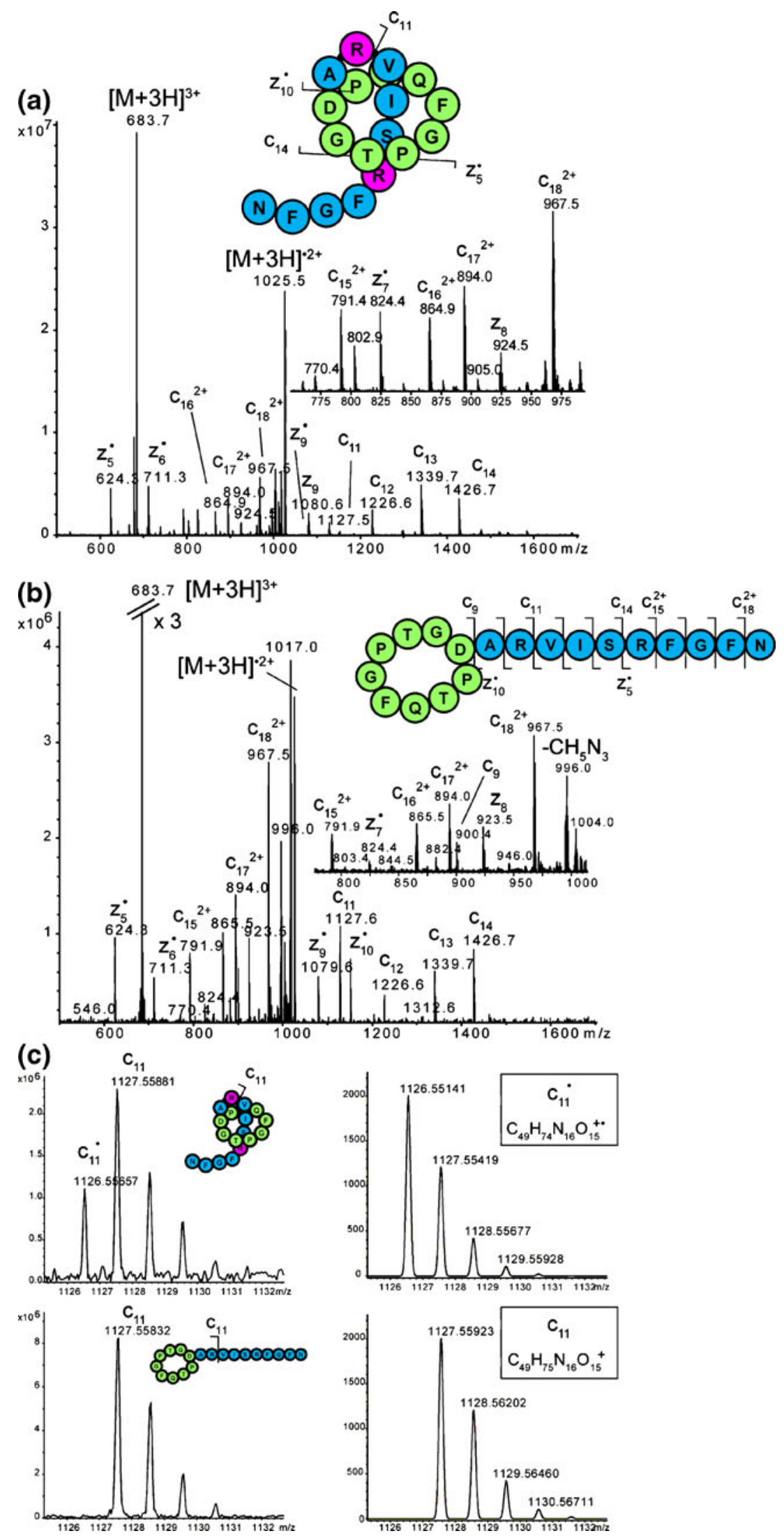

Figure 6. ECD spectra of the $[\mathrm{M}+3 \mathrm{H}]^{3+}$ species of (a) capistruin, and (b) capistruin-Icm $(\mathrm{m} / \mathrm{z} 683.7)$, recorded with a ECD bias of $1.2 \mathrm{~V}$ and an irradiation time of $100 \mathrm{~ms}$. Typical product ions are displayed; (c) $c_{11} / c_{11}$ e isotopic profiles obtained experimentally for capistruin and capistruin-Icm (on the left), and expected for $c_{11}$ and $c_{11} \bullet$ (on the right) 
The influence of conformation on the extent of hydrogen migration has been proposed from earlier studies [43, 44]. Lasso peptides constitute an attractive model to investigate this trend, given their well defined compact and stable structure. The ECD results obtained here on the two topoisomers capistruin and capistruin-lcm constitute the first direct evidence of the influence of topology on hydrogen migration, a process that was mainly interpreted with regard to amino acid sequences. It suggests potential applications in the characterization of peptide or protein conformations, topology or non-covalent associations.

\section{Acknowledgments}

The authors acknowledge support for this work by the ANR project no. BLAN_NT09_692063 and the Deutsche Forschungsgemeinschaft (DFG MA 811-25/1). They thank the TGE High field FTICR and the $\mathrm{SM}^{3} \mathrm{P}$ for access to the FTICR mass spectrometer.

\section{References}

1. Taylor, W.R.: Protein knots and fold complexity: some new twists. Comput. Biol. Chem. 31, 151-162 (2007)

2. Meluzzi, D., Smith, D.E., Arya, G.: Biophysics of Knotting. Annu. Rev. Biophys. 39, 349-366 (2010)

3. Rosengren, K.J., Craik, D.J.: How bugs make lassos. Chem. Biol. 16, 1211-1212 (2009)

4. Kolmar, H.: Biological diversity and therapeutic potential of natural and engineered cystine knot miniproteins. Curr. Opin. Pharmacol. 9, 608614 (2009)

5. Rebuffat, S., Blond, A., Destoumieux-Garzón, D., Goulard, C., Peduzzi, J.: Microcin J25, from the macrocyclic to the lasso structure: implications for biosynthetic, evolutionary, and biotechnological perspectives. Curr. Protein Pept. Sci. 5, 383-391 (2004)

6. Knappe, T.A., Linne, U., Xie, X., Marahiel, M.A.: The Glucagon Receptor Antagonist BI-32169 Constitutes a New Class of Lasso Peptides. FEBS Lett. 584, 785-789 (2010)

7. Iwatsuki, M., Tomoda, H., Uchida, R., Gouda, H., Hirono, S., Omura, S.: Lariatins, antimycobacterial peptides produced by Rhodococcus sp. K01-B0171, have a lasso structure. J. Am. Chem. Soc. 128, 7486-7491 (2006)

8. Knappe, T.A., Linne, U., Zirah, S., Rebuffat, S., Xie, X., Marahiel, M. A.: Isolation and structural characterization of capistruin, a lasso peptide predicted from the genome sequence of Burkholderia thailandensis E264. J. Am. Chem. Soc. 130, 11446-11454 (2008)

9. Tanaka, T., Tsukuda, E., Nozawa, M., Nonaka, H., Ohno, T., Kase, H., Yamada, K., Matsuda, Y.: RES-701-1, a novel, potent, endothelin type $\mathrm{B}$ receptor-selective antagonist of microbial origin. Mol. Pharmacol. 45, 724-730 (1994)

10. Salomón, R.A., Farías, R.N.: Microcin 25, a novel antimicrobial peptide produced by Escherichia coli. J. Bacteriol. 174, $7428-7435$ (1992)

11. Kimura, K., Kanou, F., Takahashi, H., Esumi, Y., Uramoto, M., Yoshihama, M.: Propeptin, a new inhibitor of prolyl endopeptidase produced by Microbispora. I. Fermentation, isolation, and biological properties. J. Antibiot. 50, 373-378 (1997)

12. Weber, W., Fischli, W., Hochuli, E., Kupfer, E., Weibel, E.K.: Anantin-a peptide antagonist of the atrial natriuretic factor (ANF). I. Producing organism, fermentation, isolation, and biological activity. J. Antibiot. 44, 164-171 (1991)

13. Destoumieux-Garzón, D., Duquesne, S., Péduzzi, J., Goulard, C., Desmadril, M., Letellier, L., Rebuffat, S., Boulanger, P.: The Ironsiderophore transporter FhuA is the receptor for the antimicrobial peptide microcin J25: role of the microcin Val11-Pro16 $\beta$-hairpin region in the recognition mechanism. Biochem. J. 389, 869-876 (2005)

14. Mukhopadhyay, J., Sineva, E., Knight, J., Levy, R.M., Ebright, R.H.: Antibacterial peptide microcin $\mathrm{J} 25$ inhibits transcription by binding within and obstructing the RNA polymerase secondary channel. Mol. Cell 14, 739-751 (2004)

15. Wilson, K.A., Kalkum, M., Ottesen, J., Yuzenkova, J., Chait, B.T., Landick, R., Muir, T., Severinov, K., Darst, S.A.: Structure of microcin $\mathrm{J} 25$, a peptide inhibitor of bacterial RNA polymerase, is a Lassoed tail. J. Am. Chem. Soc. 125, 12475-12483 (2003)

16. Rosengren, K.J., Blond, A., Afonso, C., Tabet, J.C., Rebuffat, S., Craik, D.J.: Structure of thermolysin cleaved microcin j25: extreme stability of a two-chain antimicrobial PDeptide devoid of covalent links. Biochemistry 43, 4696-4702 (2004)

17. Rosengren, K.J., Clark, R.J., Daly, N.L., Goransson, U., Jones, A., Craik, D.J.: Microcin J25 has a threaded sidechain-to-backbone ring structure and not a head-to-tail cyclized backbone. J. Am. Chem. Soc. 125, 12464-12474 (2003)

18. Loo, J.A., He, J.X., Cody, W.L.: Higher order structure in the gas phase reflects solution sStructure. J. Am. Chem. Soc. 120, 45424543 (1998)

19. Katahira, R., Shibata, K., Yamasaki, M., Matsuda, Y., Yoshida, M. Solution structure of endothelin B receptor selective antagonist RES701-1 determined by 1 H NMR spectroscopy. Bioorg. Med. Chem. 3, 1273-1280 (1995)

20. Pavlova, O., Mukhopadhyay, J., Sineva, E., Ebright, R.H., Severinov, K.: Systematic structure-activity analysis of microcin J25. J. Biol. Chem. 283, 25589-25595 (2008)

21. Knappe, T.A., Linne, U., Robbel, L., Marahiel, M.A.: Insights into the biosynthesis and stability of the Lasso peptide capistruin. Chem. Biol. 16, 1290-1298 (2009)

22. Meng, F., Forbes, A.J., Miller, L.M., Kelleher, N.L.: Detection and localization of protein modifications by high resolution tandem mass spectrometry. Mass Spectrom. Rev. 24, 126-134 (2005)

23. Senko, M.W., Speir, J.P., McLafferty, F.W.: Collisional activation of large multiply charged ions using fourier transform mass spectrometry. Anal. Chem. 66, 2801-2808 (1994)

24. Crowe, M.C., Brodbelt, J.S.: Infrared Multiphoton Dissociation (IRMPD) and collisionally activated dissociation of peptides in a quadrupole ion trap with selective IRMPD of phosphopeptides. $J$. Am. Soc. Mass Spectrom. 15, 1581-1592 (2004)

25. Kruger, N.A., Zubarev, R.A., Horn, D.M., McLafferty, F.W.: Electron capture dissociation of multiply charged peptide cations. Int. J. Mass Spectrom. 185/186/187, 787-793 (1999)

26. Bakhtiar, R., Guan, Z.: Electron capture dissociation mass spectrometry in characterization of peptides and proteins. Biotechnol. Lett. 28, 10471059 (2006)

27. Cooper, H.J., Hakansson, K., Marshall, A.G.: The role of electron capture dissociation in biomolecular analysis. Mass Spectrom. Rev. 24, 201-222 (2005)

28. Breuker, K., McLafferty, F.W.: Stepwise evolution of protein native structure with electrospray into the gas phase, $10(-12)$ to $10(2)$ s. Proc. Natl. Acad. Sci. U.S.A. 105, 18145-18152 (2008)

29. Breuker, K., Oh, H., Horn, D.M., Cerda, B.A., McLafferty, F.W.: Detailed unfolding and folding of gaseous ubiquitin ions characterized by electron capture dissociation. J. Am. Chem. Soc. 124, 6407-6420 (2002)

30. Roepstorff, P., Fohlman, J.: Proposal for a common nomenclature for sequence ions in mass spectra of peptides. Biomed. Mass Spectrom. 11, 601 (1984)

31. Biemann, K.: Appendix 5. Nomenclature for peptide fragment ions (Positive Ions). Methods Enzymol. 193, 886-887 (1990)

32. Wysocki, V.H., Tsaprailis, G., Smith, L.L., Breci, L.A.: Mobile and localized protons: a framework for understanding peptide dissociation. J. Mass Spectrom. 35, 1399-1406 (2000)

33. Leymarie, N., Costello, C.E., O'Connor, P.B.: Electron capture dissociation initiates a free radical reaction cascade. J. Am. Chem. Soc. 125, 8949-8958 (2003)

34. Cooper, H.J.: Investigation of the presence of $b$ ions in electron capture dissociation mass spectra. J. Am. Soc. Mass Spectrom. 16, 1932-1940 (2005)

35. Haselmann, K.F., Schmidt, M.: Do b-Ions occur from vibrational excitation upon $\mathrm{H}$-desorption in electron capture dissociation? Rapid Commun. Mass Spectrom. 21, 1003-1008 (2007)

36. Lee, S., Chung, G., Kim, J., Oh, H.B.: Electron capture dissociation mass spectrometry of peptide cations containing a lysine homologue: a mobile proton model for explaining the observation of b-Type product ions. Rapid Commun. Mass Spectrom. 20, 3167-3175 (2006) 
37. Liu, H., Håkansson, K.: Abundant b-Type ions produced in electron capture dissociation of peptides without basic amino acid residues. $J$. Am. Soc. Mass Spectrom. 18, 2007-2013 (2007)

38. Li, X., Lin, C., Han, L., Costello, C.E., O'Connor, P.B.: Charge remote fragmentation in electron capture and electron transfer dissociation. $J$. Am. Soc. Mass Spectrom. 21, 646-656 (2010)

39. Laskin, J., Yang, Z., Ng, C.M., Chu, I.K.: Fragmentation of $\alpha$-radical cations of arginine-containing peptides. J. Am. Soc. Mass Spectrom.21, 511-521 (2010)

40. Cooper, H.J., Hudgins, R.R., Hakansson, K., Marshall, A.G.: Characterization of amino acid side chain losses in electron capture dissociation. J. Am. Soc. Mass Spectrom. 13, 241-249 (2002)
41. Lin, C., Cournoyer, J.J., O'Connor, P.B.: Use of a double resonance electron capture dissociation experiment to probe fragment intermediate lifetimes. J. Am. Soc. Mass Spectrom. 17, 1605-1615 (2006)

42. Zhang, Z.: Prediction of electron-transfer/capture dissociation spectra of peptides. Anal. Chem. 82, 1990-2005 (2010)

43. Tsybin, Y.O., He, H., Emmett, M.R., Hendrickson, C.L., Marshall, A.G.: Ion activation in electron capture dissociation to distinguish between nterminal and C-terminal product ions. Anal. Chem. 79, 7596-7602 (2007)

44. Lin, C., Cournoyer, J.J., O'Connor, P.B.: Probing the gas-phase folding kinetics of peptide ions by IR activated DR-ECD. J. Am. Soc. Mass Spectrom. 19, 780-789 (2008) 